ARTICLE

\title{
Synchronous RNA conformational changes trigger ordered phase transitions in crystals
}

\author{
Saminathan Ramakrishnan ${ }^{1,14}$, Jason R. Stagno (1) 1,14, Chelsie E. Conrad ${ }^{1,12}$, Jienyu Ding ${ }^{1}$, Ping Yu1, \\ Yuba R. Bhandari', Yun-Tzai Lee (1) 1, Gary Pauly², Oleksandr Yefanov³, Max O. Wiedorn (1) 3, Juraj Knoska (1) 3,4, \\ Dominik Oberthür ${ }^{3}$, Thomas A. White (0) ${ }^{3}$, Anton Barty ${ }^{3}$, Valerio Mariani ${ }^{3}$, Chufeng Li ${ }^{3,5}$, Wolfgang Brehm ${ }^{3}$, \\ William F. Heinz (1) ${ }^{6}$, Valentin Magidson ${ }^{6}$, Stephen Lockett ${ }^{6}$, Mark S. Hunter (1) ${ }^{7}$, Sébastien Boutet (1) ${ }^{7}$, \\ Nadia A. Zatsepin (1) 5,13, Xiaobing Zuo (1) ${ }^{8}$, Thomas D. Grant (i) ${ }^{9}$, Suraj Pandey ${ }^{10}$, Marius Schmidt (10) ${ }^{10}$, \\ John C. H. Spence ${ }^{5}$, Henry N. Chapman (1) ${ }^{3,4,11} \&$ Yun-Xing Wang (iD ${ }^{1 凶}$
}

Time-resolved studies of biomacromolecular crystals have been limited to systems involving only minute conformational changes within the same lattice. Ligand-induced changes greater than several angstroms, however, are likely to result in solid-solid phase transitions, which require a detailed understanding of the mechanistic interplay between conformational and lattice transitions. Here we report the synchronous behavior of the adenine riboswitch aptamer RNA in crystal during ligand-triggered isothermal phase transitions. Direct visualization using polarized video microscopy and atomic force microscopy shows that the RNA molecules undergo cooperative rearrangements that maintain lattice order, whose cell parameters change distinctly as a function of time. The bulk lattice order throughout the transition is further supported by time-resolved diffraction data from crystals using an X-ray free electron laser. The synchronous molecular rearrangements in crystal provide the physical basis for studying large conformational changes using time-resolved crystallography and micro/nanocrystals.

\footnotetext{
${ }^{1}$ Structural Biophysics Laboratory, National Cancer Institute, Frederick, MD, USA. ${ }^{2}$ Chemical Biology Laboratory, Center for Cancer Research, National Cancer Institute, Frederick, MD, USA. ${ }^{3}$ Center for Free-Electron Laser Science, Deutsches Elektronen-Synchrotron DESY, Hamburg, Germany. ${ }^{4}$ Department of Physics, Universität Hamburg, Hamburg, Germany. ${ }^{5}$ Department of Physics, Arizona State University, Tempe, AZ, USA. ${ }^{6}$ Optical Microscopy and Analysis Laboratory, Cancer Research Technology Program, Frederick National Laboratory for Cancer Research, Frederick, MD, USA. ${ }^{7}$ Linac Coherent Light Source, SLAC National Accelerator Laboratory, Menlo Park, CA, USA. ${ }^{8}$ X-ray Science Division, Argonne National Laboratory, Lemont, IL, USA. ${ }^{9}$ Department of Structural Biology, Jacobs School of Medicine and Biomedical Sciences, SUNY University at Buffalo, Buffalo, NY, USA. ${ }^{10}$ Kenwood Interdisciplinary Research Complex Physics Department, University of Wisconsin-Milwaukee, Milwaukee, WI, USA. ${ }^{11}$ Centre for Ultrafast Imaging, Universität Hamburg,

Hamburg, Germany. ${ }^{12}$ Present address: Huntsman Cancer Institute, University of Utah, Salt Lake City, UT, USA. ${ }^{13}$ Present address: Department of Chemistry and Physics, ARC Centre of Excellence in Advanced Molecular Imaging, La Trobe Institute for Molecular Science, La Trobe University, Melbourne 3086 Victoria, Australia. ${ }^{14}$ These authors contributed equally: Saminathan Ramakrishnan, Jason R. Stagno. ${ }^{凶}$ email: wangyunx@mail.nih.gov
} 


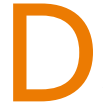
iffusionless solid-solid (SS) phase transitions, which are observed in both inorganic and organic crystals, are usually triggered by temperature, humidity, pressure, or mechanical stress that cause rearrangements of atoms or small molecules ${ }^{1,2}$. Well-known examples are shape changes in memory alloys caused by temperature variation ${ }^{3}$, or in small molecular crystals by light exposure ${ }^{4,5}$. SS phase transitions have also been observed in living systems with functional roles, for example, in strain-induced tailsheath contraction in T-even bacteriophages ${ }^{6,7}$ and in flagellaassisted bacterial locomotion ${ }^{8,9}$. Mechanisms of transition are not well understood at the atomic or molecular levels due to technical challenges ${ }^{10}$, even though they are fundamentally important to material and biological sciences.

The rapid development of the ultra-brilliant femtosecond $\mathrm{X}$ ray-free electron laser (XFEL) has made it possible to directly visualize atomic motions, ligand binding and reaction intermediates, and covalent bond breakage and formation at room temperature ${ }^{11-18}$. All those studies involved photoactive or other proteins that undergo relatively small and local structural changes within the same crystal lattices. However, the majority of biological processes involve interactions that result in large conformational changes greater than several angstroms, which are likely to alter lattice interfaces and trigger phase transitions in crystal $^{14,19-22}$. Whether lattice order is maintained throughout SS transitions is paramount to performing time-resolved crystallography (TRX) experiments on such systems.

Here we report the interplay of synchronous molecular conformational changes and ordered SS transitions in crystals of adenine riboswitch aptamer RNA (riboA), elucidated using a multidisciplinary approach. Due to lattice restraints, the conformational changes that occur in the crystal, which drive the crystal phase transition, take place on a timescale much greater than that which occur in solution or under physiological conditions. Direct visualization by polarized video microscopy (PVM) reveals both optical and physical manifestations of the SS transition in the riboA crystals in real time. Quantitative analysis of the birefringence intensity of the crystals throughout the transition allows for the elucidation of transition times and kinetics, and the level of molecular synchrony at the pixel level. Further direct visualization and image analysis at the molecular level using solution atomic force microscopy (AFM) reveal the distinct molecular arrangements and lattice constants at various stages of the transition. These microscopy data corroborate the TRX data that detect the difference electron density (DED) and evolution of intermediate structures. Together, these results describe in detail the SS transition in the riboA crystals, and demonstrate the molecular cooperativity associated with the large conformational changes triggered by ligand binding.

\section{Results and discussion}

Visualizing the SS transitions by PVM. We observed the SS transitions in small plate-like riboA crystals $\left(\sim 25 \times 25 \times 1 \mu \mathrm{m}^{3}\right)$ directly by measuring the changes in the intensity of crystal birefringence using PVM (Fig. 1a, Supplementary Fig. 1, and Supplementary Movie 1). The crystal "fractures" upon transition, suggesting a volume change. Detailed analyses were performed at the pixel level for a region of interest (ROI, Fig. 1a, yellow square) of the digitized video. The ROI, which contains $\sim 400$ million RNA molecules, is about the typical size of crystals used in the XFEL experiments ${ }^{14}$. The direct visualization at the pixel level shows that the crystal remains birefringent, indicating that the crystal never enters a liquid phase throughout the transition process. The time-traced transmitted light intensity vs. time of a single pixel exhibits multiphasic sigmoidal transitions with $\sim 50 \%$ total loss in transmitted light intensity $I_{i}\left[(x y)_{i}, t\right]$ of pixel $I(i=1,2, \ldots, 32,400)$ (Fig. 1b, left), over the course of the entire transition. The first derivatives $\left.-\partial I_{i}\left[(x y)_{i} t\right)\right] / \partial t$ give rise to the half-width and transition time (Fig. 1b, right). Three main transitions (T1, T2, and T3) are apparent, with T2 split into two sub-transitions (T2a and T2b) in terms of transition width (Fig. 1c and Supplementary Fig. 2). T1 is sharp and centered at $\sim 227 \mathrm{~s}$, with $>90 \%$ of all pixels completing transition within $5 \mathrm{~s}$ (transition width). The dependence of the transition time on the area sampling size is informative regarding the degree of spatiotemporal synchrony of a transition (Fig. 1d). The mean transition times of all three transitions appear relatively independent of the size of the sampled areas, with a standard deviation of $\sim 2.5 \mathrm{~s}$ for $\mathrm{T} 1 \mathrm{in}$ particular (Fig. 1d), indicating that the transition synchrony spans the entire sampled area (Supplementary Movie 2). The PVM result shows that the transition occurs within the ordered lattices.

Molecular order visualized using AFM. At nanometer-scale resolution, AFM can discern the ordered arrangements of individual biomacromolecules on crystal surfaces and provide the unit-cell constants. Each AFM image in Fig. 2a depicts roughly the same $50 \times 50 \mathrm{~nm}^{2}$ area of an $a c$-face riboA crystal (Supplementary Figs. 3 and 4 , see also "Methods"), equivalent to an $\sim 2 \times 2$ pixels square area in the PVM experiment. Unit-cell measurements identified three primary lattices, defined as apo unit cell (AUC), transition unit cell 1 (TUC1), and bound unit cell (BUC). We observed continuous, cooperative, and subtle variations in molecular surface topography (topo) for the time periods before the AUC to TUCl, and before the TUC1 to BUC rapid transitions. Those topographical variations are likely due to continuous molecular rotation and translation (Fig. 2a and Supplementary Table 1). The first transition, AUC to TUC1, is measurable by the change in the $c$-axis from 9.3 to $7.9 \mathrm{~nm}$ (Fig. 2b). The phase transition from TUC1 to BUC, however, is slow enough to observe an intermediate topography (Fig. 2a (image \#119), b (image $\# 119$ and \#120) and Supplementary Table 1). One portion of image \#119 (red box) corresponds to TUC1 topo 2, which is similar to the preceding AFM image; another portion (white box) corresponds to BUC topo 1, which is similar to the succeeding AFM image. A third portion (green box), which is sandwiched in between, exhibits a topography that is distinct from either (Fig. 2a), and whose autocorrelation plot gives a $c$-axis measurement of $\sim 12.5 \mathrm{~nm}$. Mixed lattices in transition (AUC and TUC1), as well as a unique lattice (TUC2) with a $c$-axis of $12.4 \mathrm{~nm}$, were observed in X-ray diffraction experiments (see the next section). Overall, the $c$-axis serves as a sensitive indicator for the multiphasic transition and is plotted vs. serial image number in real-time (Fig. 2b). The plot qualitatively parallels the PVM intensity plots (Fig. 1b). Based on the sequential appearance in both PVM and AFM, T1 observed by PVM corresponds to the AUC-TUC1 lattice transition, whereas T2 and T3 (Fig. 1c and see also Supplementary Fig. 2) are likely related to the transitions from TUC1 to TUC2, and from (TUC1/TUC2) to BUC (Fig. 2b). The AFM images and the time trace of the $c$-axis show that the crystal remains ordered throughout the transitions.

Bulk-lattice order during the transitions. The level of cooperativity (lattice order) throughout the SS phase transition was measurable by the quality and maximum scattering angle (resolution) of Bragg diffraction and by diffusive scattering that indicates deviation from perfect $\operatorname{order}^{23-25}$. Diffraction data were recorded at mixing time delays ranging from 0 to $175 \mathrm{~s}$ (Fig. 3 and see also Supplementary Fig. 5) and provide snapshots of bulk-lattice order over a large number of crystals, as opposed to the local imaging area in a single crystal shown by PVM and AFM during the transitions. With increasing delay time, the indexing rate for AUC falls off steeply with a coincident emergence of the pseudo-monoclinic transitional unit cell, TUC1 (Fig. 3a and Supplementary Table 2). Importantly, Bragg diffraction is maintained throughout the entire transition 

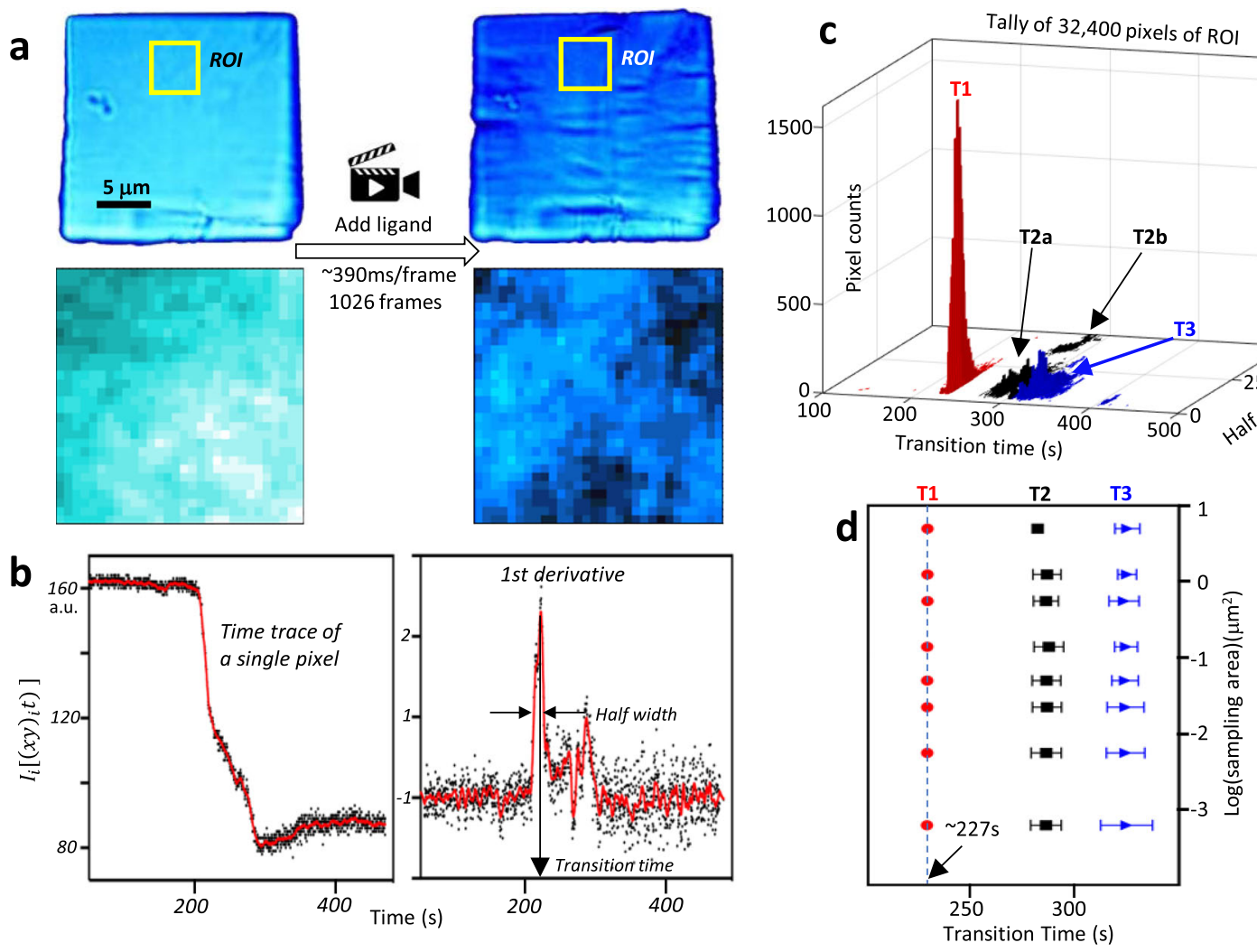

Fig. 1 Ligand-triggered crystal lattice transition observed by PVM. a Video microscopy images of an "ab" (c-axis along the thin edge) riboA crystal before (top left) and after (top right) the phase transition in the presence of $1 \mathrm{mM}$ ligand. The yellow box defines the $\sim 4.5 \mu \mathrm{m}$ square $(180 \times 180=32,400$ pixels) region of interest (ROI) used for the detailed analysis. The crystal is continuously monitored at the pixel level, starting from the beginning (bottom left) to the end (bottom right). A $30 \times 30$ pixels square area in the yellow box is used to show the crystal is birefringent at the pixel level before (bottom left) and after (bottom right) the transition. The images are color-enhanced for clear visualization of individual pixels and the nonuniform crystal surface. $\mathbf{b}$ Intensity $I_{i}\left[(x y)_{i} t\right]$ (arbitrary units) vs. time trace (in s) for the single-center pixel of the ROI. Black dots represent the intensities of individual pixels, and the red line is a time average of the derivative with a smoothing window of $15.2 \mathrm{~s}$ (left). Derivative $\left.-\partial l_{i}\left[(x y)_{i} t\right)\right] / \partial t$ vs. time so that peaks represent the maximum rate of intensity decrease (right). Black dots represent the instantaneous derivative at each time point. The red line is a time average of the derivative with a smoothing window of $7.78 \mathrm{~s}$. c Bivariate histogram of peak half-width of transitions 1, 2, and 3 (T1, T2a and b, and T3) ( $y$-axis) and transition time ( $x$-axis) for all $(32,400)$ pixels in the ROI. d Transition time vs. size of sampling areas. Time zero in the PVM experiment refers to the first frame (200) used in the digitization. Source data are provided as a Source Data file.

from AUC to TUC1, with very little change in the diffraction hitrate (Fig. 3a), and no evidence of crystals becoming completely disordered or entering the liquid phase. AUC-indexed diffraction data for each time delay (observable up to $75 \mathrm{~s}$ ) were assembled and aligned in three-dimensional space ("3D merged") according to orientation information provided by indexing (Fig. $3 b)^{23,26}$. In the $a^{*} b^{*}$ plane, only Bragg peaks corresponding to AUC dimensions of $a(48.3 \AA)$ and $b(46.9 \AA)$ are observed at $0 \mathrm{~s}$. At $10 \mathrm{~s}$, an additional set of Bragg peaks emerges corresponding to $a(50.3 \AA)$ and $b$ (25.2 $\AA$ ) dimensions of TUC1, which become more prominent with increasing delay time. The presence of peaks corresponding to TUC1 in the $3 \mathrm{D}$ reciprocal lattices merged with respect to AUCindexed orientations indicates that the TUC1 diffraction comes from AUC-indexed and oriented crystals (Fig. 3b). Upon manual inspection of individual diffraction patterns, numerous images exhibited such differentiated AUC and TUC1 lattices in the same crystals (Fig. 3c), illustrating the sudden yet cooperative behavior of T1, corroborating the PVM (Fig. 1) and AFM (Fig. 2) results. In the $a^{*} c^{*}$ plane, the rapid sampling by TRX reveals azimuthal streaks about $b^{*}$ at $10 \mathrm{~s}$, whose degree of elongation increases at $25 \mathrm{~s}$ (Fig. 3b), and coincides with the shortening of the $c$-axis (from 93.4 to $78.7 \AA$ ) and the change in the unique angle of the monoclinic cell (from $\beta$ to $\alpha$ ) (Fig. 3c). The fact that radially elongated peaks are not observed in $a^{*} b^{*}$ suggests that these features are not the result of crystal mosaicity in the conventional sense. Instead, the molecular disorder occurs anisotropically-order is retained to a greater extent along certain directions-and is determined intrinsically by the trajectory of molecular motion and the effects it has on crystal packing. Such lattice restructuring includes both the disruption of some packing interactions and the formation of new ones, which are not necessarily isotropic or uniform in all directions.

The lattice parameters derived from the diffraction data correlate well with those measured from time-lapsed AFM data (Fig. $2 \mathrm{~b}$ and Supplementary Table 1). In particular, the $c$-axis measurement $(\sim 12.5 \mathrm{~nm})$ observed in the TUC1-BUC intermediate (Fig. 2a, image \#119) was used to guide the indexing of diffraction data. TUC2, with a $c$-axis of $12.4 \mathrm{~nm}$, was identified in a small population (500 crystals) of the mixing data (up to $175 \mathrm{~s}$ ), and in a greater population (3770 crystals) in a separate pool of data collected from sample premixed with ligand ( $>7 \mathrm{~min}$ post mixing) (Supplementary Tables 3 and 4).

Implications of synchronous phase transitions. The ordered lattices during the transition make it possible to record large molecular conformational changes in real time. Structural changes associated with the AUC to TUC1 transition were observed in DED maps generated from AUC ligand-free (AUC-free), AUC 10 

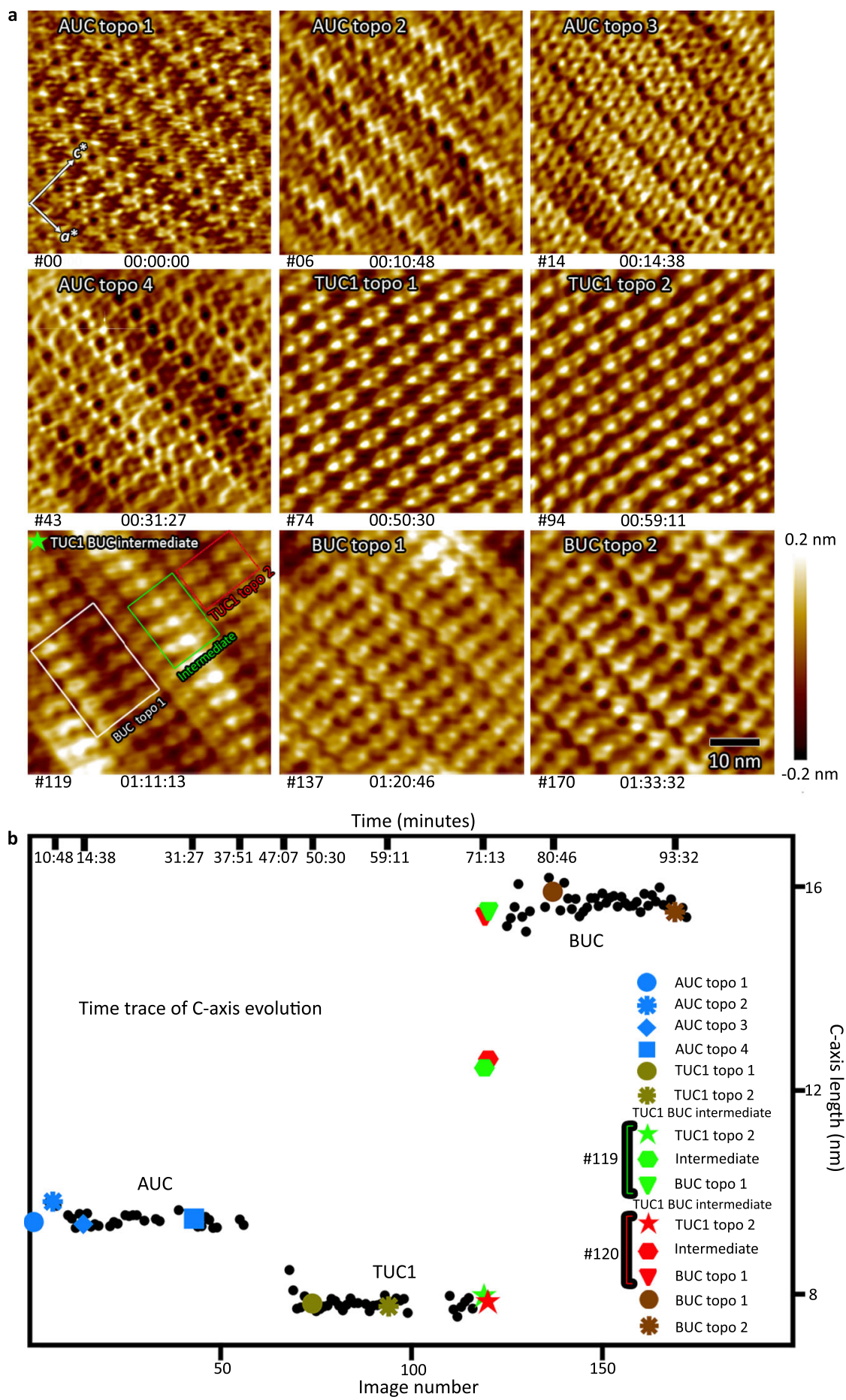

Fig. 2 Ligand-triggered crystal lattice transition observed by AFM. a The time-lapsed representative snapshots of the ligand-induced phase transitions in ac face of the riboA crystal. AUC apo unit cell, TUC1 transition unit cell 1, TUC2 transition unit cell 2, BUC bound unit cell. The order of morphological changes and phase transitions is as follows: AUC topography (topo) 1, AUC topo 2, AUC topo 3, AUC topo 4, TUC1 topo 1, TUC1 topo 2, TUC1-BUC intermediate, BUC topo 1, and BUC topo 2. $\mathbf{b}$ The plot of $c$-axis length in nanometers vs. serial image number and time (top axis) during the lattice transition. This plot summarizes the ligand-triggered polymorphic transition. Gaps in the plot are the periods when the changes in the crystal phase resulted in the loss of contact with the crystal surface during AFM imaging. Source data are provided as a Source Data file.

s-mixed (AUC-10s), and AUC $25 \mathrm{~s}$-mixed (AUC-25s) data sets (Fig. 4 and Supplementary Table 5). For both molecules in the asymmetric unit (apo1 and apo2), large changes relative to the starting apo conformations are observed at $10 \mathrm{~s}$ (Fig. $4 \mathrm{a}$, b, left panels). Changes in apol are primarily localized to the top of the $5^{\prime}$ strand of P1, beginning at G18, and extending through the flexible hinge region that includes A21, U22, and A23 (Fig. 4a, left). These changes likely reflect the conversion of apol (ligand incompetent) toward apo2 (ligand competent), which involves large motions in the hinge and $\mathrm{P} 1$, including an $\sim 180^{\circ}$ rotation of $\mathrm{A} 23$ out of the binding pocket. Between 10 and $25 \mathrm{~s}$, fewer changes are observed in apo1, although prominent peaks appear near latch residues 47-49, as well as G44 (Fig. 4a, right), and could be further indicative of changes toward the apo 2 conformation. At $10 \mathrm{~s}$, the 

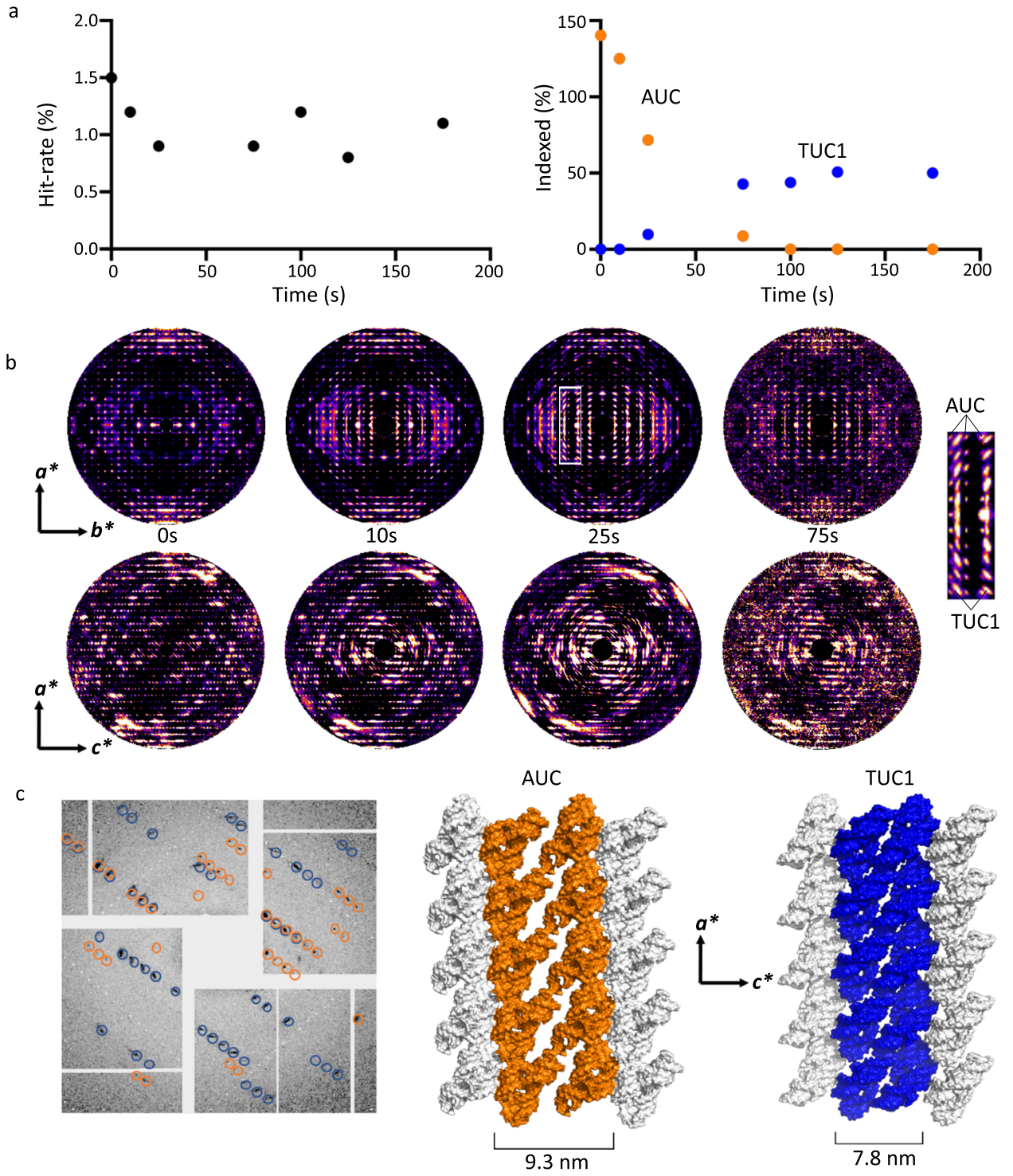

Fig. 3 Phase transition observed by time-resolved serial crystallography. a Plots of X-ray diffraction hit-rate (left) and indexing rate (right) for AUC (orange) and TUC1 (blue) lattices as a function of delay time after mixing 1:1 with $20 \mathrm{mM}$ ADE. For the non-mixed sample (0 s), the average ratio of sample:buffer was 2:1, yielding a slightly higher hit-rate. The AUC to TUC1 conversion occurs much earlier than that observed in PVM and AFM experiments, where lower ligand concentrations ( $1 \mathrm{mM}$ for PVM, and 50 or $150 \mu \mathrm{M}$ for AFM) were used due to the requirements of those experiments. b Cross-sections $\left(a^{\star} b^{\star}, a^{\star} c^{\star}\right.$ ) of the 3D-merged reciprocal lattices of AUC-indexed crystals at different delay times. An expanded subsection (white box) of $a^{\star} b^{\star}$ at 25 s shows the emergence of broad, intense Bragg peaks corresponding to the $b$-axis (25.3 $\AA$ ) of TUC1. c Left: single diffraction image from a single crystal with circled indexed Bragg reflections belonging to either AUC (orange) or TUC1 (blue). Changes in crystal packing upon conversion from AUC (middle) to TUC1 (right), resulting in a shortening of the c-axis from 9.3 to $7.9 \mathrm{~nm}$ and a change in the $\beta$-angle from $94.5^{\circ}$ to $90.6^{\circ}$.

population of DED peaks (particularly positive ones) is much greater for apo2 (Fig. 4b, left). The differences are primarily clustered at the bottom of the three-way junction, specifically latch residues 46-48, hinge residues $21-23$, and junction residues $73-74$ that connect P3 and P1.

The conversion of apo2 to IB.Ade, in which U48 is displaced by $\mathrm{Ade}^{14}$, is strongly supported by the large (4.5 sigma) negative DED peak between U48's uracil base and sugar moiety. The further conversion of IB.Ade to B.Ade-like is impeded by the lattice constraint between the latch region in $\mathrm{P} 2$ and the P3-kissing loop of an adjacent symmetry molecule, and serves as the primary ratelimiting factor in the phase transition from AUC to TUC1. A21 also restricts the exit of U48 (latch) from the binding pocket. A21 is part of the flexible hinge, and ultimately forms one of three base triples that stabilize P1 in the B·Ade-like configuration. Together, the constraints on IB.Ade in the AUC lattice limit molecular motion for quite some time, as minimal differences are observed between 10 and $25 \mathrm{~s}$ (Fig. 4b, right). The DED at G44, however, may reflect disruption of the dominant P2-P2 crystal packing interface in AUC (Fig. 4b). With highly redundant sets of diffraction data, at multiple time points during the AUC-TUC1 transition, we expect that the large conformational changes can be time-resolved crystallographically 27 and that transitional structures could be further constrained by an analysis of the diffusive scattering ${ }^{23}$.

Using a combination of methods-PVM, AFM, and XFEL-the results of this study demonstrate, in real time, the synchrony of molecular changes that occur in the ligand-induced phase transition of riboA crystals (Supplementary Table 6). We also performed a similar PVM experiment using a caged adenine compound 
10 s - free

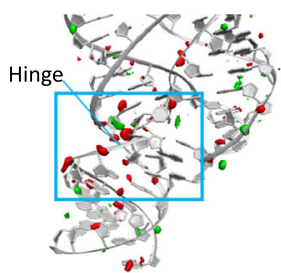

$10 \mathrm{~s}-\mathrm{fre}$

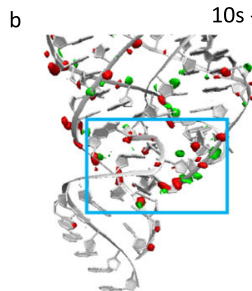

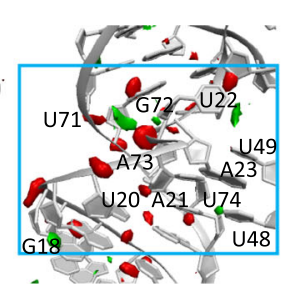

ree
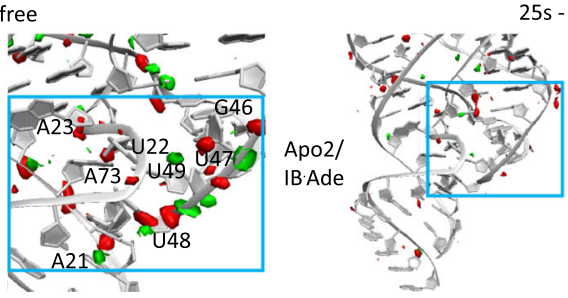

$25 s-10 s$
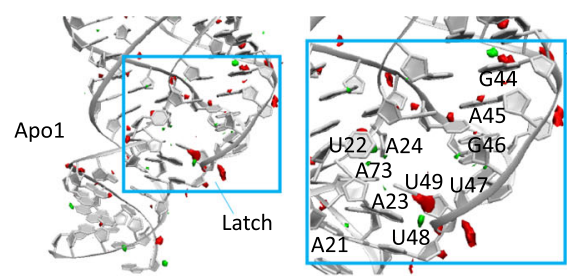

$25 s-10 s$

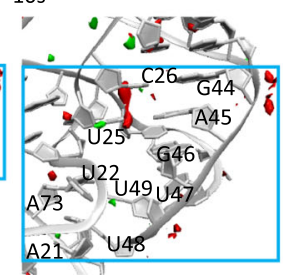

Fig. 4 Difference electron density reflecting early stages of AUC-TUC1 transition. $F_{\mathrm{o}}-F_{\mathrm{o}}$ difference electron density maps, displayed for apo1 (a) and apo2 (b), generated using the ligand-free model (PDB: 5E54) and the observed structure factor differences between data sets. Left: AUC-10 s-AUC free; right: AUC-25 s-AUC-10 s. Positive and negative density are shown in green and red, respectively, contoured at 3 sigma.

(Supplementary Fig. 6 and see also "Methods"), whereby the reaction of ligand binding was triggered by photoactivation at $365 \mathrm{~nm}$ (Supplementary Movie 3). Such experiments present an alternative approach to achieve potentially faster and more uniform reaction induction, particularly in cases where diffusive mixing is problematic. The synchronized SS transition in the riboA crystals is not unique, as revealed by PVM experiments using crystals of the tetracycline riboswitch aptamer (Supplementary Fig. 7). Therefore, the types of approaches presented here may prove highly useful for time-resolved studies of other biomolecular systems involving large conformational changes or crystal phase transitions.

\section{Methods}

RNA sample preparations. Detailed experimental procedures for the in vitro transcription, purification, characterization, and crystallization of the adenine riboswitch aptamer RNA have been reported previously ${ }^{14,28}$. Crystallization buffer was prepared as $40 \mathrm{mM}$ sodium cacodylate, $80 \mathrm{mM} \mathrm{KCl}, 100 \mathrm{mM} \mathrm{MgCl}, 12 \mathrm{mM}$ spermine tetrahydrochloride, and 35-65\% (v/v) 2-methyl-2,4-pentanediol (MPD). The stabilization buffer (SB) was prepared by mixing an equal volume of crystallization buffer and RNA buffer (RB: $10 \mathrm{mM}$ HEPES pH 7.5, $10 \mathrm{mM} \mathrm{KCl}, 0.5 \mathrm{mM}$ EDTA). All solutions were mixed to homogeneity prior to use, and stored at room temperature.

Synthesis and characterization of the caged adenine $\mathbf{N}$-(2-nitrobenzyl)-9Hpurine-6-amine. Photo-caged adenine (pcADE) was prepared as follows: 6-chloropurine $(4.10 \mathrm{~g}, 26.5 \mathrm{mmol}$ ) and 2-nitrobenzylamine hydrochloride $(5.00 \mathrm{~g}, 26.5 \mathrm{mmol})$ were suspended in $100 \mathrm{ml}$ of isopropanol. Triethylamine $(5.37 \mathrm{~g}, 53.0 \mathrm{mmol})$ was added and the reaction was warmed in a $90{ }^{\circ} \mathrm{C}$ oil bath. After $2 \mathrm{~h}$, the temperature was reduced to $80^{\circ} \mathrm{C}$ and the reaction was stirred for an additional $16 \mathrm{~h}$. A dense precipitate formed during that time. The suspension was chilled on ice for $1 \mathrm{~h}$ and filtered. The filtrate was washed with several portions of cold isopropanol and dried under high vacuum to give $3.28 \mathrm{~g}$ (12.1 mmol, 45.7\%) of $\mathrm{N}$-(2-nitrobenzyl)- $9 H$-purine-6-amine. Proton nuclear magnetic resonance $\left({ }^{1} \mathrm{H}\right.$ NMR) $\left(\mathrm{DMSO}-d_{6}\right) \delta=12.99(\mathrm{~s}, 1 \mathrm{H}, \mathrm{H})$, 8.28 (br s, $1 \mathrm{H}, \mathrm{H}), 8.16-8.14(\mathrm{~m}, 2 \mathrm{H}, \mathrm{H}), 8.04(\mathrm{~d}, J=8.1 \mathrm{~Hz}, 1 \mathrm{H}, \mathrm{H}), 7.66$ (t, $J=7.6$ $\mathrm{Hz}, 1 \mathrm{H}, \mathrm{H}), 7.57(\mathrm{~d}, J=6.8 \mathrm{~Hz}, 1 \mathrm{H}, \mathrm{H}), 7.50(\mathrm{t}, J=7.7 \mathrm{~Hz}, 1 \mathrm{H}, \mathrm{H}), 4.98(\mathrm{~s}, 2 \mathrm{H}, \mathrm{H}) .{ }^{13} \mathrm{C}$ NMR (DMSO- $d_{6}$ ) $\delta=154.1$ (br), 152.3, 149.8 (br), 148.1, 139.3, 135.2 (br), 133.7, $129.9,128.9,124.5,118.9$ (br), 40.3 (br). MS (atmospheric pressure chemical ionization (APCI) $) \mathrm{m} / z=271.1[\mathrm{M}+\mathrm{H}]^{+}$.

NMR spectra were recorded on a Bruker spectrometer at $500 \mathrm{MHz}$ for ${ }^{1} \mathrm{H}$ and $126 \mathrm{MHz}$ for ${ }^{13} \mathrm{C}$. The following abbreviations were utilized to describe peak patterns: $\mathrm{br}=$ broad, $\mathrm{s}=$ singlet, $\mathrm{d}=$ doublet, $\mathrm{t}=$ triplet, and $\mathrm{m}=$ multiplet. All chemicals were obtained from Sigma-Aldrich and used without further purification. The identity and homogeneity of pcADE were assessed with a single quadrupole LC/MSD (Agilent) equipped with an in-line diode-array ultraviolet (UV) detector. Samples were injected onto a Zorbax rapid-resolution reversedphase C18 column (bore size: $100 \times 2.1 \mathrm{~mm}^{2}$; particle size: $3.5 \mu \mathrm{m}$ ), coupled with a $\mathrm{C} 18$ guard column $\left(12.5 \times 2.1 \mathrm{~mm}^{2}\right)$. The contents were eluted at a flow rate of $300 \mu \mathrm{L} / \mathrm{min}$ with a $5-90 \%$ gradient of methanol/water containing $0.1 \%$ acetic acid. Samples were analyzed using APCI. Full scan mass spectra, as well as the total-ion chromatogram and the UV chromatogram at $270 \mathrm{~nm}$, were used to assess compound purity.
Polarized video microscopy. In PVM, the polarization angle of linearly polarized light changes as it passes through a birefringent sample such as a crystal. An analyzer polarizer placed in front of a camera selects a single polarization angle for detection. Spatial and temporal differences in birefringence are detected as changes in transmitted light intensity at the camera, thus providing direct observation of the optical effects induced by the lattice transitions (Fig. 1). In order to perform a PVM experiment, we had to overcome the problem of crystal motion after mixing with the ligand, caused by turbulence as well as crystal shape changes. We tested several compounds to optimize crystal adhesion to glass surfaces for imaging. Initially, crystals grown by batch crystallization methods were harvested, adsorbed on glassbottom dishes treated with poly-D-lysine, Cell-Tak, or just plasma cleaned, and then used for imaging. Ultimately, mica-grown crystals adhered to poly-D-lysinecoated glass were used for the final PVM experiments (discussed below in detail).

Supplementary Figure 1a illustrates the setup of the PVM. We modified a Nikon Eclipse TE200 from a surplus. The setup consists of a frame and base of a Nikon Eclipse TE200 microscope equipped with a halogen lamp light source for transillumination. Nikon microscope objectives $\times 100 / 1.45$ Plan Apo Lambda and $\times 20 / 0.75$ Plan Apo were used with a NA 0.72 condenser. Videos were recorded on a C-mounted OMAX color CMOS camera (Model A35180U3, AmScope, Irvine, $\mathrm{CA})$ with $18 \mathrm{MP}$ pixel resolution and the provided Touplite software that interfaces with the camera. A fixed polarizer in the "emitter" position functioned as an analyzer in the polarization setup. To implement computer-controlled rotatable polarizer, motorized rotational stage (Thorlabs, product \# KPRM1E) was positioned above the condenser. Prior to the time-lapse imaging, fields of riboswitch crystals were identified by eye, and the linear polarizer was rotated to maximize the transmitted intensity of a majority of crystals in the field. Imaging was performed with the $\times 100$ objective.

The adenine ligand was introduced to the crystal using two complementary methods. In the diffusion method, crystals grown on mica were extracted with $5 \mu \mathrm{L}$ $\mathrm{SB}$ and deposited onto a poly-D-lysine-coated glass-bottom dish (MatTek Corporation, P35GC-1.5-14-C) (Supplementary Fig. 1b), and then covered with $1.5 \mathrm{~mL} \mathrm{SB}$. A measure of $1.5 \mathrm{~mL}$ of adenine $(2 \mathrm{mM})$ in $\mathrm{SB}$ was added to a final concentration of $1 \mathrm{mM}$ and video of the crystal was recorded (Supplementary Movie 1) with a $200 \mathrm{~ms}$ exposure time. A $30 \times 30$ pixel area in the yellow square box (Fig. 1a) before (left) and after (right) the phase transition is shown in Supplementary Fig. 1c. The pixel resolution of these oversampled images is $24.75 \mathrm{~nm}$, but the theoretical diffraction limit for the system is $210 \mathrm{~nm}$, or an area equivalent to $\sim 9 \times 9$ pixels. The images are color-enhanced for clear visualization of individual pixels and the nonuniform crystal surface.

For the photoactivated experiments, a Thorlabs high-power UV LED at $365 \mathrm{~nm}$ (ThorLabs, Model SOLIS-365C) light source was installed at the backport of the microscope. The LED was powered by a Thorlabs driver (ThorLabs, Model DC2200) for epi-illumination with a UV beam. The filter cube contains a DAPI dichroic mirror, which directed UV-led light from the epi-port to the sample for the photoactivated experiments. Mica-grown crystals were extracted with $5 \mu \mathrm{L}$ SB, and then deposited onto a glass-bottom dish. Two hundred microliters of $200 \mu \mathrm{L}$ of freshly prepared pcADE ( $10 \mathrm{mM}$, dissolved in SB) was added to the crystals, and the glass-bottom portion of the dish was sealed with a greased coverslip (Supplementary Fig. 1b). The crystal was exposed with a 2-s LED pulse (4.5 A) of $\mathrm{UV}(365 \mathrm{~nm})$ light, and the phase transition was recorded with an exposure time of $200 \mathrm{~ms}$ (Supplementary Movie 3).

Analysis of polarization microscopy time-lapsed data. Time-lapse polarization microscopy data were processed and analyzed using custom Matlab (ver. 2019b, 
Mathworks) routines to identify when crystal lattice transitions occurred after ligand addition (or uncaging) and the speed of the transitions. The time-lapsed data consisted of a series of RGB images collected over the courses of the transitions and saved as an AVI file. Raw RGB AVI files from the polarization microscopy system were converted to greyscale uncompressed AVI files. The pixel resolution of the images is $24.75 \mathrm{~nm}$, but the theoretical diffraction limit for the system $\Delta x=\lambda / 2 \mathrm{NA}$, where $\lambda$ is the wavelength of light (taken as $500 \mathrm{~nm}$ ) and NA is the numerical aperture of the objective (1.45 for the system used here), is $172 \mathrm{~nm}$. $I(x, y, t)$ traces were smoothed in time using a sliding window of $15.2 \mathrm{~s}$ (39 time steps), prior to the derivative calculation. For the analysis presented here, a ROI of 180 pixels square $\left(4.455 \mu \mathrm{m}^{2}\right)$ was selected. We examined the effect of spatially resampling the ROI at each time point using sampling areas of $1,3,6,9,15,30,45$, and 90 pixels square resulting in ROIs with $180,60,30,20,12,6,4$, and 2 "superpixels" per side. A sampling area of 9 pixels corresponds to $225 \mathrm{~nm}$, slightly greater than the theoretical diffraction limit for the polarization video microscope.

The recorded intensity for a given $(x, y)$ pixel at time $t, I(x, y, t)$, depends on the birefringence at that pixel relative to $t=0$. For the crystals, $I(x, y, 0)$ is a maximum, and $I(x, y, t)$ generally decreases as the crystal lattice undergoes conformational rearrangements and phase transitions that change the birefringence at each pixel. To identify phase transitions (sharp decreases in the $\partial I(x, y, t) / \partial t)$, we calculated the first derivative of the intensity vs. time, $\partial I(x, y, t) / \partial t)$. $\partial I(x, y, t) / \partial t)$ has local minima at the transitions, and we multiplied $\partial I(x, y, t) / \partial t)$ by -1 to make the data compatible with peak-finding algorithms that search for maxima in Matlab library. We identified and fit peaks in $-\partial I(x, y, t) / \partial t)$ using the findpeaksxw.m routine (Pragmatic Introduction to Signal Processing Applications in scientific measurement, Tom O'Haver, March 2020 edition) with the following parameters: slope threshold, 0.00001; amplitude threshold, 0.6; smooth width, 50; fit width, 39; smooth type, 3; peak group, 2. Peak positions in time correspond to the transition times, and the peak half-widths at halfmaxes correspond to the transition durations. For each sampling area size, transition times and durations were calculated for all superpixels in the ROI, and a $k$-means algorithm was used to classify the peaks into the clusters representing the three transitions in the $I(x, y, t)$ curves. Transition times and durations were averaged and plotted against sampling area to demonstrate scale invariance of the transition characteristics. The time where $\left.\partial I_{i}\left[(x y)_{i} t\right)\right] / \partial t=0$, the peak of the derivative for each pixel, is taken as the transition time.

Each peak in $\left.-\partial I_{i}\left[(x y)_{i} t\right)\right] / \partial t$ was fit to a Gaussian. $K$-means clustering was used to identify three clusters (red, black, and blue) corresponding to the three main transitions in the crystal, namely, T1, T2 $(a$ and $b)$, and T3. The time-lapsed video of the first transition was based on the ROI resampled with the 9 pixels square sampling area. For the video, the intensities for each pixel were normalized such that the minimum value was set to zero, and the maximum was normalized to the value at $I(x, y, t=0)$. A false-color colormap was used to highlight the transitions. The $-(\partial I(x, y, t) / \partial t)$ frames are a binary representation of the transition with pixels with $-(\partial I(x, y, t) / \partial t)>$ amplitude threshold colored in red, and all others in blue. Sampling areas of $1,3,6,9,15,30,45$, and 90 pixels square were used to spatially average "blocks" of the ROI. For each sampling area size, $k$-means clusters of peak half-width durations were calculated and averaged. Red, black, and blue circles correspond to first, second, and third transitions. Data are plotted mean \pm standard deviation.

Crystal growth on mica. The AFM experiments required the riboA crystals to remain stationary throughout the imaging of the crystal before, during, and after the infusion of ligand and the lattice transition. DNA and RNA are routinely imaged with an AFM after absorption to mica treated with amino silanes. Mica is a standard, atomically flat AFM substrate with a silanol surface chemistry similar to glass. The electrostatic attraction between the negatively charged phosphate backbones of the nucleic acids and the positively charged amino groups of the substrate pins the molecules to the substrate. We reasoned that the riboswitch crystals would have negative surface charge density and that the crystals would likewise adhere electrostatically to a positively charged surface.

For the AFM experiments, we used mica treated with 3-aminopropylsilatrane (APS) as a positively charged substrate. APS-mica is a common solid surface for nucleic acid imaging by AFM because it has a lower surface roughness than mica treated with silanes such as (3-aminopropyl) triethoxysilane), which crosslink in addition to covalently binding to mica ${ }^{29}$. Due to the insufficient adsorption (possibly due to the crystal faces not being atomically flat and parallel to the mica surface across the entire crystal) and the physical changes in the crystal during the phase transition often desorbed the crystal from the mica surface. Moreover, the infusion of ligand solution through perfusion tubes creates a strong shear force on the crystals on the mica surface, which desorbs crystals. To overcome these two main issues, riboA crystals were grown directly on V1-grade mica surface by the hanging-drop vapor diffusion method. Mica itself is perfectly flat, pristine crystal free of inclusions or other defects, which may facilitate the riboA crystal growth evenly on mica surfaces by providing highly effective nucleation sites. We use seed crystals to initiate a large flat crystal growth on mica. To effectively absorb the crystal seeds on the surface, freshly peeled mica surfaces were first treated with $50 \mu \mathrm{L}$ of $100 \mathrm{mM}$ spermidine for $15 \mathrm{~min}$ at room temperature. After surface treatment, the excess of spermidine on the mica surface was washed out with plenty of distilled water and air-dried. The spermidine-treated mica samples were then fixed on EasyXtal crystallization supports (Qiagen, Germantown, MD) using paraffin oil. A measure of $0.5 \mu \mathrm{L}$ of 1:1000 dilution of riboA crystal seed stock was carefully placed on treated mica and incubated for $30 \mathrm{~s}$. Without drying the seed solution, $5 \mu \mathrm{L}$ of an equal volume of $7.5 \mathrm{~g} / \mathrm{L}$ gel-purified riboA in RNA buffer and $35 \%$ MPD crystallization buffer mixture was gently placed on the seed solution on mica samples. The support containing the crystallization sample solution was inverted and screwed onto the EasyXtal crystallization plate, followed by incubation at $22^{\circ} \mathrm{C}$ for $12 \mathrm{~h}$

We grew crystals on the mica surface with two different concentrations of seed solutions to obtain $a b$ - or $a c$-face crystals. For growing the rectangular-shaped $a c$ face crystals, a 1:1000 dilution of riboA crystal seeds was used. For square-shaped crystals with $a b$ face, a 1:100 dilution of seeds was used (Supplementary Fig. 3).

AFM imaging. The crystals were imaged in SB containing 35\% MPD with a Cypher VRS AFM outfitted with a temperature-controlled stage and perfusion cantilever holder (Asylum Research, Oxford Instruments, Santa Barbara, CA, USA) in intermittent contact mode using BL-AC40TS-C2 "BioLever mini" cantilevers (nominal tip radius of $8 \mathrm{~nm}$, spring constant of $0.09 \mathrm{~N} / \mathrm{m}$ and resonance frequency in $\mathrm{SB}$ of $\sim 12.5 \mathrm{kHz})$ at $15^{\circ} \mathrm{C}$. These levers have a high sensitivity $(100 \mathrm{~nm} / \mathrm{V})$ and low applied forces during imaging, which is essential for imaging soft biological crystals. A V1-grade mica sample with crystals was first fixed on an AFM sample holder disc (Ted Pella, Redding, CA, USA) using epoxy resin. Twenty microliters of SB was placed on the mica-crystal sample to avoid drying during fixation and covered tightly. The cantilever holder was connected with perfusion tubing to inlet and outlet liquid reservoirs ( $1 \mathrm{~mL}$ syringes) of SB to maintain the fluid volume $(100 \mu \mathrm{L})$ and for buffer exchanges during measurement. After placing the sample on the AFM stage, the thermal peak of the cantilever in SB was generated and used to determine the resonance frequency $(\sim 12 \mathrm{kHz})$. The AFM imaging temperature was set at $15^{\circ} \mathrm{C}$, where the crystals were stable to sustain high-frequency scanning by the AFM tips. All images were recorded at a scan rate of $12 \mathrm{~Hz}, 256$ pixels in $x$ and $y$, and $100-\mathrm{nm}$ scan size. First-order flattening was applied line-by-line during scanning to remove sample tilt artifacts. At $100-\mathrm{nm}$ scan size, scan rates $>12 \mathrm{~Hz}$ with 256 points and lines are not optimal for continuous recording because of tipinduced damage to the crystal surface. Scan sizes of $>100 \mathrm{~nm}$ can also be used with higher scan rates. But for the high-quality crystal surface topography, a frame size $<100 \mathrm{~nm}$ is recommended because the image quality has a direct impact on unitcell parameters derivation.

Before the addition of ligand, the apo lattice was imaged in SB continuously for at least 25-50 frames to determine the quality of the cantilever and crystal lattice. For continuous imaging, a crystal with a sizeable flat surface in the central region was chosen. A ligand-containing SB in a syringe containing $150 \mu \mathrm{M}$ (for $a c$ face) or $50 \mu \mathrm{M}$ (for $a b$ face) adenine ligand was slowly infused into the chamber while the AFM images recorded continuously. Immediately after the infusion of ligand, thermal and mechanic disturbances, results of mixing and external force from infusion, rendered image recording impossible for the first few minutes. This is one of the other reasons that the low temperature $\left(15^{\circ} \mathrm{C}\right)$ and ligand concentrations $(150$ or $50 \mu \mathrm{M})$ were chosen to slow down the transitions. For all experiments, the AFM images were recorded until the crystal lattice transition reached the final bound state, with gaps where the cantilever contact with the crystal lattice was lost, especially at the time when the crystal underwent significant physical changes. Nevertheless, the scanner position for imaging was unchanged throughout the experiment. Whenever the cantilever lost contact with the crystal surface, the imaging was stopped, and the tip approached the crystals again in its original assumed position to continue recording the transition. The most important experimental part is to use already standardized imaging parameters whenever the contact is lost. In addition, the orientation angle of crystal lattice from apo to the bound state was identical throughout the experiment, which is evident in the coordinated phase transition. Sometimes, due to the viscous nature of SB, the cantilever loosens from its original position, which may give rise to slightly tilted image acquisition. The drastic physical changes during phase transition could also result in images slightly offset from the original position. However, they do not impact the final results presented in the manuscript. In addition, to minimize damages to crystals or the cantilever tips, especially during the phase transition stages where the crystal undergoes drastic $z$-axis changes, we used the intermittent contact mode for imaging. In general, careful consideration was also given to the balance among various factors, such as recording of high-quality topographic images while minimizing damages to the lattice surface or cantilever tips throughout the experiments.

AFM of reverse transition. In addition, we conducted experiments with $50 \mu \mathrm{M}$ ligand using $a c$-face crystals, and recorded the phase transitions in both the forward and reverse directions. Once the crystal lattice transition reached BUC, the reversal of the phase transition was achieved by removing the ligand from the crystals through extensive buffer exchanges in situ. This was achieved by replacing the buffer with a ligand-free buffer ten times $(1 \mathrm{~mL}$ each) and left overnight in the AFM chamber at $15^{\circ} \mathrm{C}$ along with the cantilever on the same spot where the bound state was imaged. After overnight night soaking, the ligand-free buffer was infused and exchanged with fresh buffer $(1 \mathrm{~mL}$ each) every $15 \mathrm{~min}$ for at least another $5 \mathrm{~h}$. At the end of extensive buffer exchanges, the reversed apo lattice was recorded. To minimize evaporation of the buffer during overnight soaking, the temperature was kept at $15^{\circ} \mathrm{C}$. 
AFM image processing. The AFM images were processed using Scanning Probe Image Processor software (Image Metrology, Lyngby, Denmark). The digital resolution of images was set to 2024 pixels. The raw images were processed using either the fast Fourier transform (FFT) or the correlation averaging utilities to remove random noise. When FFT was used, the final images were calculated using the inverse FFT utility. The autocorrelation images were calculated either from the raw images directly or autocorrelated averaged images. The unit-cell dimensions were determined manually by choosing the most prominent peaks from the autocorrelation images in the contour mode to avoid miscalculation from automated analysis. For the images containing the mixed-cell lattice, the unit-cell parameters were measured directly from the images. All AFM images were processed and cropped into $50 \times 50 \mathrm{~nm}^{2}$ from their original size $\left(100 \times 100 \mathrm{~nm}^{2}\right)$.

RiboA batch crystallization. RiboA microcrystal preparation for XFEL experiments was similar to that described in ref. ${ }^{14}$. Briefly, purified riboA ( $5 \mathrm{~g} / \mathrm{L}$ in RB) was mixed to homogeneity with an equal volume of $\mathrm{CB}$. The mixture was then inoculated $(3 \% \mathrm{v} / \mathrm{v})$ with a concentrated crystal seed stock, and the crystals were grown for $2 \mathrm{~h}$ at room temperature $\left(\sim 22^{\circ} \mathrm{C}\right)$ with constant gentle mixing. The plate-like crystals $(<5 \mu \mathrm{m})$ were centrifuged at $1000 \times g$. The supernatant was removed, and the crystals were washed with SB. The crystals were centrifuged and washed again, and finally concentrated to a maximum density of $10^{9}-10^{10}$ crystals/ $\mathrm{mL}$. The fine granular precipitate, which forms during crystallization, could not be completely removed, even upon filtering $(10 \mu \mathrm{m})$, making it difficult to maximize the density of the crystal slurry without impeding/clogging sample injection. This is most likely the cause for low overall hit rates $(\sim 1 \%)$.

Diffusive mixing experiments with liquid injection. Technical details of the mixing experiments have been described previously ${ }^{14}$. Additional data were collected at the Coherent X-ray Imaging endstation ${ }^{30}$ at the Linac Coherent Light Source (LCLS) during beam times LP58 and LM95. The riboA crystal slurry was filtered through a $20-\mu \mathrm{m}$ wire-mesh filter immediately prior to loading into a stainless-steel reservoir for sample injection, which was then placed in an anti-settling device. The sample reservoir, as well as large $(10 \mathrm{~mL}$ ) reservoirs containing DEPC (diethyl pyrocarbonate)-treated water, SB, or SB with $20 \mathrm{mM}$ adenine ligand, was connected via switchyards to one of two high-performance liquid chromatography (HPLC) pumps. Reservoirs containing sample, SB, or water were connected to HPLC1/switchyard1. Reservoirs containing ligand, SB, or water were connected to HPLC2/switchyard2. The injectors were inserted into the vacuum chamber using the "nozzle rod" system ${ }^{31}$. Diffusive mixing with liquid injection was achieved using high-resolution 3D-printed microfluidic mixers and nozzles integrated upstream of the X-ray interaction region. Our miniaturized adaptation of the static Kenics mixer Design 10, with $200 \mu \mathrm{m}$ inner diameter and five helical elements for laminar stirring, provided complete mixing in $<100 \mathrm{~ms}$ for 1:1 ratio of sample and ligand flowrates ${ }^{24}$. The mixed sample was fed via a delay capillary to the double-flow focusing nozzle ${ }^{25}$ Design $8^{24}$ with an additional outer-sheath flow of ethanol that improves jet stability and prevents icing. The mixing delay, which is the time between a crystal's exposure to ligand and its exposure to $\mathrm{X}$-rays, was fully controllable through accurate placement (distance) of the mixers, and the combined flowrates (velocity), which were monitored by flowmeters. The time of diffusion, which likely occurs on the millisecond timescale ${ }^{32}$, is considered negligible.

XFEL data processing. All XFEL data were acquired at the LCLS with an X-ray photon energy of $\sim 9.5 \mathrm{keV}$, pulse energy of $\sim 3.2 \mathrm{~mJ}$, pulse length of $\sim 30 \mathrm{fs}$, and repetition rate of $120 \mathrm{~Hz}$. Data were recorded on a Cornell-SLAC Pixel Array Detector with live diffraction feedback using OnDA ${ }^{33}$. Cheetah and CrystFEL $(v 0.9 .0+b 5 d e 2753)$ were used for hit finding and indexing of diffraction images, respectively ${ }^{34-38}$. Detector geometry was optimized using CrystFEL geoptimiser ${ }^{39}$. The peakfinder 8 algorithm in CrystFEL indexamajig was used to identify Bragg reflections, with a pixel value threshold of 150 , a minimum of 2 pixels per peak and minimum $\mathrm{SNR}=4$. The diffraction patterns were indexed and integrated using CrystFEL indexamajig, using XGANDALF ${ }^{26}$, MOSFLM, ASDF, and DirAx indexing algorithms with "low-tolerance" (LT) parameters: peak-finding SNR $=4$ and unitcell tolerances of $7 \%$ for cell axes and $3^{\circ}$ for cell angles ${ }^{35,36}$. Finally, the data sets were combined into a single stream file, and the merged and scaled with two iterations in CrystFEL partialator. The partialities for scaling were fixed to a value of 1 .

Plotting indexing rates for AUC and TUC1 as a function of delay time reveals complementary sigmoidal curves: the decrease in AUC-indexed crystals is accompanied by an increase in TUC1-indexed crystals (Fig. 3a and Supplementary Fig. 5). The curves in Fig. 3a resemble the birefringence intensity curves from the PVM experiments (Fig. 1b). By $75 \mathrm{~s}$, the vast majority of crystals index as TUC1, whose indexing rate plateaus at $\sim 50 \%$. From this analysis, it is clear that the decline in total "indexability" of crystals is correlated with the lattice transition driven by riboA conformational changes upon ligand binding. Importantly, however, the hitrate remains fairly constant $(\sim 1 \%)$, indicating that Bragg diffraction is maintained throughout the transition, and that the crystals never enter a liquid or disordered phase. To further investigate the indexability of the data from 25 to $175 \mathrm{~s}$ post mixing, diffraction patterns that could not be indexed as TUC1 with LT parameters were "reindexed" with XGANDALF using "high-tolerance" (HT) parameters: peak-finding SNR $=3.5$ and unit-cell tolerances of $20 \%$ for cell axes and $10^{\circ}$ for cell angles (Supplementary Fig. 5 and Supplementary Table 3).

3D merging. Diffraction patterns for individual data sets were oriented and assembled in $3 \mathrm{D}$ reciprocal space according to unit-cell parameters using the index and orientation information provided by CrystFEL indexing algorithms ${ }^{26,36}$. Slices of the 3D reciprocal lattice were processed in FIJI ${ }^{40}$ and are displayed in Fig. 3. Changes in the AUC-indexed diffraction patterns, particularly at low resolution, were observable as early as $10 \mathrm{~s}$, and became more prominent at $25 \mathrm{~s}$. 3D-merged patterns at $75 \mathrm{~s}$ exhibited similar trends as those at $25 \mathrm{~s}$, but were difficult to characterize with so few patterns $(<2000)$ that could be indexed as AUC at $75 \mathrm{~s}$. In $a^{*} b^{*}$, a set of intense elongated peaks emerges, corresponding to the TUC1 lattice ( $a=50.3 \AA$ and $b=25.2 \AA$ ), indicating that a significant portion of single crystals exhibits both AUC and TUC1 diffraction. In the $a^{*} c^{*}$ plane, the marginal increase in the length of the $a$-axis was barely detectable, whereas the dramatic decrease in the $c$-axis (from 93.4 to $78.7 \AA$ ) and change in the beta-angle (from 94.5 to 90.6 ) was observed as azimuthal streaks about $b^{*}$.

DED map calculations. Ligand-free and $10 \mathrm{~s}$ mixed data from our previously reported work ${ }^{14}$ were reindexed using the same version of CrystFEL and indexing parameters as used for the data in the current study, yielding 29,052 and 23,786 indexed patterns, respectively (Supplementary Table 5). Those $10 \mathrm{~s}$ mixed data were then combined with the $10 \mathrm{~s}$ mixed data (14,321 indexed patterns) from the current experiment. The three data sets-AUC free (29,052 patterns), "combined" AUC-10 s (38,107 patterns), and AUC-25 s (10,357 patterns)-were merged and scaled in CrystFEL partialator using the custom-split option to ensure all data were put on the same scale before exporting the final reflection files for individual data sets. $F_{\mathrm{o}}-F_{\mathrm{o}}$ maps (Fig. 4 ) were generated with the PHENIX software suite ${ }^{41}$ using the ligand-free model for phase calculations. Since the phosphate groups are more electron-rich, the majority of DED peaks reside along the RNA backbone. Overall, the net DED appears to be negative (more red than green), indicating that, in general, the transitioning molecules become less ordered, that is, the positive DED is dispersed. However, DED is more balanced for apo2, which exhibits more prominent positive peaks (Fig. $4 \mathrm{~b}$ ).

Unlike the binding pocket, there is minimal restriction on $\mathrm{P} 1$ mobility, which has been observed previously to have incomplete electron density and significantly elevated $B$-factors even in a ligand-free environment, and is particularly worse for the apo 2 conformer ${ }^{14}$. The paucity of DED peaks in the P1 of apo2 (Fig. $4 \mathrm{~b}$ ) suggests that the regulatory helix is very dynamic at the onset of conformational switching. The mobility of residues A21, U22, and A23 in the hinge region, directly adjacent to the top of the P1 stem, most likely enable the release of ligand-induced energetic strain and partial unwinding of the helix. The combined motion in the three-way junction, however, propagates throughout the $\mathrm{P} 3$ helix and the $3^{\prime}$ strand of P1, observed as negative DED peaks (Fig. 4b). We suspect, therefore, that the displacement of $\mathrm{U} 48$, which affects the bottom of $\mathrm{P} 3$ via the three-way junction and the top of P3 via lattice interactions, may temporarily alter the stability of both $\mathrm{P} 1$ and $\mathrm{P} 3$, as the molecule transitions to B.Ade-like.

A similar analysis was done by comparing TUC1-indexed data from 25 to $75 \mathrm{~s}$ with that of 100 to $175 \mathrm{~s}$. However, no discernable differences in electron density between the two data sets were observed. This further supports that the bulk conformational changes in reaching a B-Ade-like state may occur in the context of the AUC lattice. However, AUC would seem to require enough expansion to overcome lattice restraints inhibiting reconfiguration of the binding pocketthereby enabling rotation and translation of the P1 helices, as both molecules coordinate to stabilize into the lower-energy TUC1 lattice. As a result, structure determination of intermediate conformers in the trajectories from apol to apo2, or IB.Ade to B.Ade-like, may be feasible by sorting of structure factor amplitudes and their associated phases through singular value decomposition ${ }^{42}$.

Structure determination and refinement. Molecular replacement (MR) and model refinement were performed using the PHENIX software suite ${ }^{41}$. All structure analysis and modeling were done in Coot ${ }^{43}$. Images containing structural models were generated using The PyMOL Molecular Graphics System (Schrödinger, LLC). The crystal structures derived from TUC1-indexed (6VWT) or TUC2-indexed (6VWV) data were solved by MR using the riboA bound structure (RCSB: 4TZX) as a search model. Crystal data and refinement statistics are summarized in Supplementary Table 4.

\section{Data availability}

Structure factors and model coordinates for the crystal structures of TUC1 and TUC2 are available from the PDB under accession codes 6VWT and 6VWV, respectively. Data regarding AFM and PVM experiments are available within the manuscript. Source data are provided with this paper.

\section{Code availability}

The code for the analyses of PVM data is available for download from the GitHub site with link: https://github.com/Will-Heinz/OMAL-PVM-Analyzer. 
Received: 29 July 2020; Accepted: 2 December 2020;

Published online: 19 March 2021

\section{References}

1. Commins, P., Desta, I. T., Karothu, D. P., Panda, M. K. \& Naumov, P. Crystals on the move: mechanical effects in dynamic solids. Chem. Commun. 52, 13941-13954 (2016).

2. Anwar, J. \& Zahn, D. Polymorphic phase transitions: macroscopic theory and molecular simulation. Adv. Drug Deliv. Rev. 117, 47-70 (2017)

3. Otsuka, K. \& Wayman, C. M. Shape Memory Materials (Cambridge Press, 1998).

4. Etter, M. C. \& Siedle, A. R. Solid-State rearrangement of (phenylazophenyl) palladium hexafluoroacetylacetonate. J. Am. Chem. Soc. 105, 641-643 (1983).

5. Skoko, Z., Zamir, S., Naumov, P. \& Bernstein, J. The thermosalient phenomenon. "Jumping Crystals" and crystal chemistry of the anticholinergic agent oxitropium bromide. J. Am. Chem. Soc. 132, 14191-14202 (2010).

6. Olson, G. B. \& Hartman, H. Martensite and life-displacive transformations as biological processes. J. Phys. 43, 855-865 (1982).

7. Harris, W. F. \& Scriven, L. E. Cylindrical crystals, contractile mechanisms of bacteriophages and the possible role of dislocations in contraction. J. Theor. Biol. 27, 233-257 (1970).

8. Baker, M. D., Wolanin, P. M. \& Stock, J. B. Signal transduction in bacterial chemotaxis. Bioessays 28, 9-22 (2006).

9. Calladine, C. R. Change of waveform in bacterial flagella: the role of mechanics at the molecular level. J. Mol. Biol. 118, 22 (1978).

10. Mnyukh, Y. Fundamentals of Solid-State Phase Transitions, Ferromagnetism and Ferroelectricity (DirectScientific Press, 2009).

11. Tenboer, J. et al. Time-resolved serial crystallography captures high-resolution intermediates of photoactive yellow protein. Science 346, 1242-1246 (2014).

12. Olmos, J. L. Jr. et al. Enzyme intermediates captured "on the fly" by mix-andinject serial crystallography. BMC Biol. 16, 59 (2018).

13. Barends, T. R. et al. Direct observation of ultrafast collective motions in CO myoglobin upon ligand dissociation. Science 350, 445-450 (2015).

14. Stagno, J. R. et al. Structures of riboswitch RNA reaction states by mix-andinject XFEL serial crystallography. Nature 541, 242-246 (2017).

15. Pande, K. et al. Femtosecond structural dynamics drives the trans/cis isomerization in photoactive yellow protein. Science 352, 725-729 (2016)

16. Nango, E. et al. A three-dimensional movie of structural changes in bacteriorhodopsin. Science 354, 1552-1557 (2016).

17. Nogly, P. et al. Retinal isomerization in bacteriorhodopsin captured by a femtosecond X-ray laser. Science 361, https://doi.org/10.1126/science.aat0094 (2018).

18. Kim, K. H. et al. Direct observation of bond formation in solution with femtosecond X-ray scattering. Nature 518, 385-389 (2015).

19. Dunitz, J. D. Phase transitions in molecular crystals: looking backwards, glancing sideways. Phys Scr. 91, https://doi.org/10.1088/0031-8949/91/11/ 112501 (2016)

20. Harata, K. \& Akiba, T. Structural phase transition of monoclinic crystals of hen egg-white lysozyme. Acta Crystallogr. D 62, 375-382 (2006).

21. Svanidze, A. V., Huth, H., Lushnikov, S. G., Kojima, S. \& Schick, C. Phase transition in tetragonal hen egg-white lysozyme crystals. Appl. Phys. Lett. 95, https://doi.org/10.1063/1.3275858 (2009).

22. Vijayalakshmi, L., Krishna, R., Sankaranarayanan, R. \& Vijayan, M. An asymmetric dimer of beta-lactoglobulin in a low humidity crystal formstructural changes that accompany partial dehydration and protein action. Proteins 71, 241-249 (2008).

23. Ayyer, K. et al. Macromolecular diffractive imaging using imperfect crystals. Nature 530, 202-+ (2016).

24. Knoska, J. et al. Ultracompact 3D microfluidics for time-resolved structural biology. Nat. Commun. 11, 657 (2020).

25. Oberthuer, D. et al. Double-flow focused liquid injector for efficient seria femtosecond crystallography. Sci. Rep. 7, 44628 (2017).

26. Yefanov, O. et al. Mapping the continuous reciprocal space intensity distribution of X-ray serial crystallography. Philos. Trans. R. Soc. Ser. B 369, https://doi.org/10.1098/rstb.2013.0333 (2014).

27. Neutze, R. Opportunities and challenges for time-resolved studies of protein structural dynamics at X-ray free-electron lasers. Philos. Trans. R. Soc. Lond. Ser. B 369, 20130318 (2014).

28. Stagno, J. R., Bhandari, Y. R., Conrad, C. E., Liu, Y. \& Wang, Y. X. Real-time crystallographic studies of the adenine riboswitch using an $\mathrm{X}$-ray free-electron laser. FEBS J. 284, 3374-3380 (2017).

29. Lyubchenko, Y. L., Shlyakhtenko, L. S. \& Ando, T. Imaging of nucleic acids with atomic force microscopy. Methods 54, 274-283 (2011).

30. Liang, M. N. et al. The coherent X-ray imaging instrument at the Linac coherent light source. J. Synchrotron Radiat. 22, 514-519 (2015).
31. Weierstall, U., Spence, J. C. H. \& Doak, R. B. Injector for scattering measurements on fully solvated biospecies. Rev. Sci. Instrum. 83, https://doi. org/10.1063/1.3693040 (2012).

32. Schmidt, M. Mix and inject: reaction initiation by diffusion for time-resolved macromolecular crystallography. Adv. Condens. Matter Phys. 2013, 1-10 (2013).

33. Mariani, V. et al. OnDA: online data analysis and feedback for serial X-ray imaging. J. Appl Crystallogr. 49, 1073-1080 (2016).

34. Barty, A. et al. Cheetah: software for high-throughput reduction and analysis of serial femtosecond X-ray diffraction data. J. Appl. Crystallogr. 47, 1118-1131 (2014)

35. White, T. A. et al. Crystallographic data processing for free-electron laser sources. Acta Crystallogr. D 69, 1231-1240 (2013).

36. White, T. A. et al. CrystFEL: a software suite for snapshot serial crystallography. J. Appl. Crystallogr. 45, 335-341 (2012).

37. White, T. A. Processing serial crystallography data with CrystFEL: a step-bystep guide. Acta Crystallogr. D 75, 219-233 (2019).

38. White, T. A. et al. Recent developments in CrystFEL. J. Appl. Crystallogr. 49, 680-689 (2016).

39. Yefanov, O. et al. Accurate determination of segmented X-ray detector geometry. Opt. Express 23, 28459-28470 (2015).

40. Schindelin, J. et al. Fiji: an open-source platform for biological-image analysis. Nat. Methods 9, 676-682 (2012).

41. Liebschner, D. et al. Macromolecular structure determination using X-rays, neutrons and electrons: recent developments in Phenix. Acta Crystallogr. D 75, 861-877 (2019).

42. Schmidt, M., Rajagopal, S., Ren, Z. \& Moffat, K. Application of singular value decomposition to the analysis of time-resolved macromolecular X-ray data. Biophys. J. 84, 2112-2129 (2003).

43. Emsley, P., Lohkamp, B., Scott, W. G. \& Cowtan, K. Features and development of Coot. Acta Crystallogr. D 66, 486-501 (2010).

\section{Acknowledgements}

This work was supported in part by the Intramural Research Program of the National Cancer Institute, National Institutes of Health (Y.-X.W.), the NSF-STC “BioXFEL” (NSF1231306) (J.C.H.S.), the Maxwell computational resources operated at Deutsches Elektronen-Synchrotron (DESY) (H.N.C.), Hamburg, Germany (H.N.C.), and NSF Award (NSF-1565180) (N.A.Z.). Use of the Linac Coherent Light Source (LCLS), SLAC National Accelerator Laboratory, is supported by the US Department of Energy, Office of Science, Office of Basic Energy Sciences under Contract No. DE-AC02-76SF00515 (S.B.) Parts of the sample injector used at LCLS for this research were funded by the National Institutes of Health, P41GM103393, formerly P41RR001209.

\section{Author contributions}

Y.-X.W. conceptualized and designed the study; P.Y. and J.R.S. provided the RNA samples; P.Y., and J.D. characterized RNA samples; S.R. and J.R.S. crystallized the RNA; J.R.S., C.E.C., Y.R.B., O.Y., M.O.W., J.K., D.O., T.A.W., A.B., V.Mariani, C.L., W.B., M.S.H., S.B., S.P., M.S., T.D.G., N.A.Z., and Y.-X.W. performed TRX experiments and analysis of diffraction data; S.R., J.R.S., W.F.H., V.Magidson, S.L., and Y.-X.W. performed PVM experiments. W.F.H. wrote the MATLAB codes for the PVM analysis. Y.-X.W. W.F.H., S.R., J.R.S., and Y.-X.W. analyzed PVM data. S.R., W.F.H., and J.D. performed AFM experiments. Y.-T.L. provided tetracycline riboswitch data. G.P. synthesized caged adenine. Y.-X.W. and X.Z. performed kinetics analyses. J.C.H.S. and H.N.C. provided XFEL expertise and support. S.R., J.R.S., and Y.-X.W. drafted the manuscript and all authors contributed to the revision.

\section{Funding}

Open Access funding provided by the National Institutes of Health (NIH).

\section{Competing interests}

The authors declare no competing interests.

\section{Additional information}

Supplementary information The online version contains supplementary material available at https://doi.org/10.1038/s41467-021-21838-5.

Correspondence and requests for materials should be addressed to Y.-X.W.

Peer review information Nature Communications thanks the anonymous reviewer(s) for their contribution to the peer review of this work. Peer reviewer reports are available.

Reprints and permission information is available at http://www.nature.com/reprints

Publisher's note Springer Nature remains neutral with regard to jurisdictional claims in published maps and institutional affiliations. 
(c) (i) Open Access This article is licensed under a Creative Commons Attribution 4.0 International License, which permits use, sharing, adaptation, distribution and reproduction in any medium or format, as long as you give appropriate credit to the original author(s) and the source, provide a link to the Creative Commons license, and indicate if changes were made. The images or other third party material in this article are included in the article's Creative Commons license, unless indicated otherwise in a credit line to the material. If material is not included in the article's Creative Commons license and your intended use is not permitted by statutory regulation or exceeds the permitted use, you will need to obtain permission directly from the copyright holder. To view a copy of this license, visit http://creativecommons.org/ licenses/by/4.0/.

This is a U.S. government work and not under copyright protection in the U.S.; foreign copyright protection may apply 2021 